\title{
Dwarf galaxies and the Magnetisation of the IGM
}

\author{
Uli Klein ${ }^{1}$ \\ ${ }^{1}$ Argelander-Institut für Astronomie, \\ Auf dem Hügel 71, Bonn, Germany \\ email: uklein@astro.uni-bonn.de
}

\begin{abstract}
With the operation of LOFAR, a great opportunity exists to shed light on a problem of some cosmological significance. Diffuse radio synchrotron emission not associated to any obvious discrete sources as well as Faraday rotation in clusters of galaxies both indicate that the intergalactic or intracluster medium (IGM, ICM) is pervaded by a weak magnetic field, along with a population of relativistic particles. Both, particles and fields must have been injected into the IGM either by Active Galactic Nuclei (AGN) or by normal star-forming galaxies. Excellent candidates for the latter are starburst dwarf galaxies, which in the framework of hierarchical structure formation must have been around in large numbers. If this is true, one should be able to detect extended synchrotron halos of formerly highly relativistic particles around local starburst or post-starburst dwarf galaxies. With LOFAR, one should easily find these out to the Coma Cluster and beyond.
\end{abstract}

Keywords. acceleration of particles, magnetic fields, polarization, radiation mechanisms: nonthermal, ISM: jets and outflows, ISM: magnetic fields, galaxies: clusters: general, galaxies: dwarf, galaxies: starburst, radio continuum: galaxies

\section{Introduction}

Dwarf galaxies play a key role in the enrichment of the ICM or IGM, not only as far as heavy elements are concerned, but possibly also regarding the magnetisation. According to the standard bottom-up scenario of galaxy formation, primeval galaxies must have injected much of their (enriched) interstellar medium (ISM) into the IGM during the initial bursts of star formation, thereby "polluting" large volumes of intergalactic space because of their high number density. By the same token, the following two properties render dwarf galaxies potentially very efficient in injecting a relativistic plasma into their surroundings: first, they exist in large numbers, and second, they possess low escape velocities, making it easier to expel their interstellar gas, as compared to massive spiral galaxies. Such galactic winds are in fact seen in some prototypical low-mass galaxies in the local universe.

\section{Outflows and the magnetisation of galaxy clusters}

Galaxy clusters are known to be pervaded by a relativistic plasma, i.e. particles with (mildly) relativistic energy and magnetic fields. In the centres of some rich clusters, radio halos have been found, produced by either primary or secondary electrons (see Fig. 1, left). The problem with primary electrons is their relatively short lifetime, limited by 

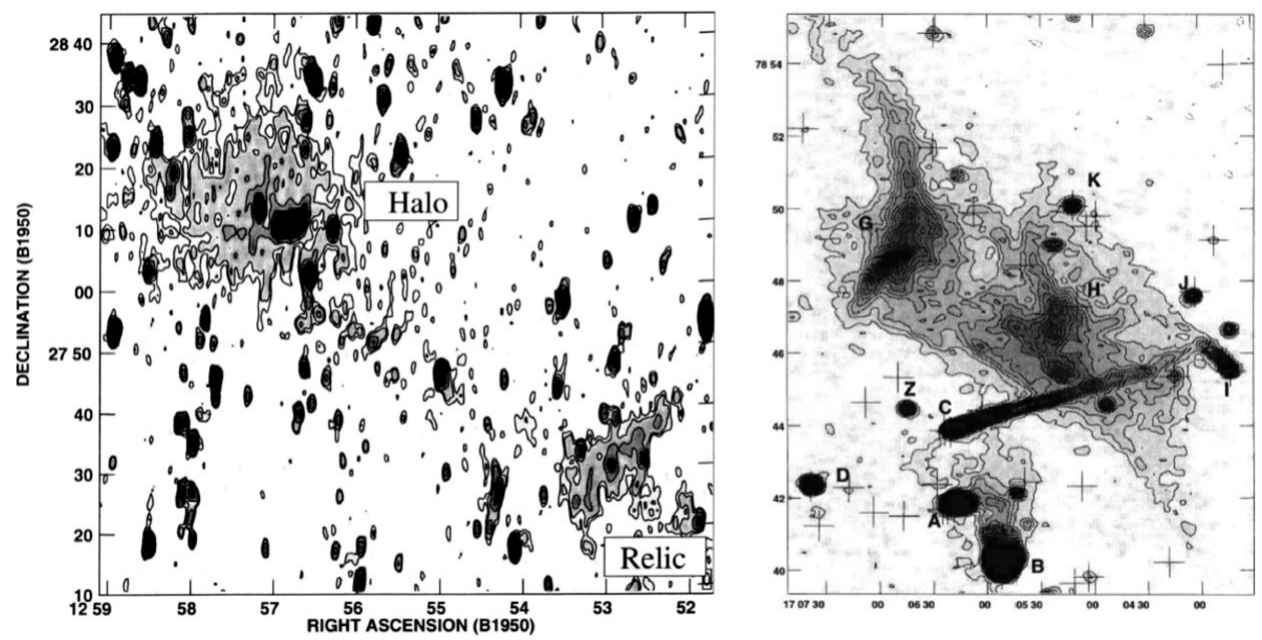

Figure 1. Diffuse radio continuum emission from clusters of galaxies: central radio halo and peripheral structure in the Coma Cluster (left, from Giovannini et al. 1993), and radio relic in A 2556 (right, from Röttgering et al. 1994).

synchrotron and inverse-Compton losses:

$$
t_{1 / 2}=1.59 \cdot 10^{9} \cdot \frac{B^{1 / 2}}{B^{2}+B_{c m b}^{2}}\left[\left(\frac{\nu}{\mathrm{GHz}}\right)(1+z)\right]^{-1 / 2},
$$

where

$$
B_{c m b}=(1+z)^{2} \mu \mathrm{G}
$$

is the equivalent magnetic field strength of the cosmic microwave background. This latter equation results from the similar dependences of synchrotron and inverse-Compton losses,

$$
\begin{aligned}
& \dot{E}_{\text {synch }} \propto-B^{2} \cdot E^{2} \\
& \dot{E}_{I C} \propto-u_{\text {rad }} \cdot E^{2},
\end{aligned}
$$

where $u_{\text {rad }}$ is the energy density of the radiation field (which for the perfect black-body CMB radiation is $\propto T_{c m b}^{4}$ ). Eq. (2.1) then tells us that, for instance, with a magnetic field of $B=1 \mu \mathrm{G}$, particles radiating at $1.4 \mathrm{GHz}$ will be rendered invisible after $t_{1 / 2}=10^{8} \mathrm{yr}$ in the local universe $(z=0)$. Hence, primary electrons require continuous injection. However, diffuse radio halos are never associated with any obvious "fresh" source, and one therefore has to invoke secondary electrons. These are produced by hadronic collisions of relativistic protons with the thermal gas in a pion-muon chain. Owing to their much larger mass, protons have synchrotron lifetimes exceeding a Hubble time. Finally, it should be pointed out that completely independent evidence for the magnetisation of (at least the) central regions of galaxy clusters comes from the observation of Faraday rotation (e.g. Clarke et al. 2001).

Clusters undergoing large-scale merging frequently exhibit so-called radio relics, mostly on their periphery (Fig. 1, right). In contrast to radio halos, these are significantly polarised, probably reflecting magnetic-field enhancement in the compression zones where the subclusters produce large-scale shockwaves during their mutual penetration. Naturally, this also provides an efficient acceleration mechanism. Particles that were formerly highly relativistic (electrons in particular) have cooled via synchrotron and 
inverse-Compton losses. During a cluster merger, they are boosted to high energies again, and the acceleration regions shine up in the radio regime.

Kronberg et al. (1999) were the first to raise the question whether low-mass galaxies could have made a significant contribution to the magnetisation of the IGM (apart from more massive starburst galaxies and AGN). Owing to their large number (observed and predicted in a CDM cosmology) and their injection of relativistic particles as described above, they could have played a cardinal role in the context of this cosmologically important scenario. If true, it is to be expected that dwarf galaxies are "wrapped" in large envelopes of previously highly relativistic particles - and magnetic fields, which are pushed out of them during epochs of vigorous star formation. Bertone et al. (2006) have discussed this more quantitatively and made predictions for the strengths of magnetic seed fields, to be then amplified by large-scale dynamos over cosmic time. In particular, they also predict the existence of magnetic voids.

Of course, in magnetising the ICM/IGM, low-mass galaxies have been competing with AGN. Judging from the radio luminosities of the "culprits", it is clear that nevertheless low-mass galaxies may have contributed significantly. A typical starburst dwarf galaxy emits a monochromatic radio luminosity of $P_{1.4 \mathrm{GHz}} \approx 10^{20.5} \mathrm{~W} \mathrm{~Hz}^{-1}$, while this figure is $P_{1.4 \mathrm{GHz}} \approx 10^{24.7} \mathrm{~W} \mathrm{~Hz}^{-1}$ for radio galaxies in the $\mathrm{FRI} / \mathrm{II}$ transition regime. Hence, the radio power produced by AGN is some 15000 times larger than that of dwarf galaxies. However, $(\Lambda \mathrm{CDM})$ cosmology helps at this point, since dwarf galaxies have come in huge numbers if the bottom-up scenario of structure formation holds. Furthermore, the lifetime of radio galaxies is limited (Bird et al. 2008), $\tau_{l i f e} \approx 1.5 \cdot 10^{7} \mathrm{yr}$, with duty cycles of $\tau_{\text {life }} \approx 8 \cdot 10^{8} \mathrm{yr}$. Hence, this yields an effective activity period of $\tau_{\text {active }} \approx 2 \cdot 10^{8} \mathrm{yr} \approx$ $0.015 \times \tau_{\text {Hubble }}$.

Finally, it should be noted that central so-called "mini-halos", which are bright extended radio sources located in the centres of cooling-flow clusters (Perseus A, Hydra A, Virgo A) cannot do the magnetisation job: they are pressure-confined.

\section{Local templates}

Measurements of the radio continuum radiation of dwarf galaxies over a large frequency range have shown that ongoing star formation in them is accompanied by enhanced radio continuum emission (Klein 1982; Klein et al. 1991). Owing to their shallow gravitational potentials, the containment of relativistic cosmic-ray particles in such galaxies is, however, low, as inferred from studies of the spectral index and magnetic-field structure. Fig. 2 demonstrates this: in contrast to massive spiral galaxies, whose radio continuum is dominated by synchrotron radiation at cm wavelengths (Gioia et al. 1982) there is a lack of it for dwarf galaxies. The lack is more pronounced the lower the luminosity (hence mass) of the dwarf galaxy (Klein et al. 1991). These relativistic particles streaming into the halos of dwarf galaxies lose their energy on a time scale of $\sim 10^{8}$ yr according to Eq. (2.1) via synchrotron and Inverse-Compton radiation. They are thus quickly rendered invisible at $\mathrm{cm}$ wavelengths, while their synchrotron emission will still be detectable at metre waves where their lifetime is 5 to 10 times longer.

The existence of winds in low-mass galaxies is inferred from the observed kinematics of the gas (measured with slit spectroscopy), but can arguably be also inferred from measurements of the temperature of the hot (X-ray-emitting) gas. For instance, Martin $(1998,1999)$ found the outflow velocities in NGC 1569 to exceed the escape speed, and della Ceca et al. (1996) derived a temperature of its hot, X-ray-emitting gas to exceed the virial temperature. 

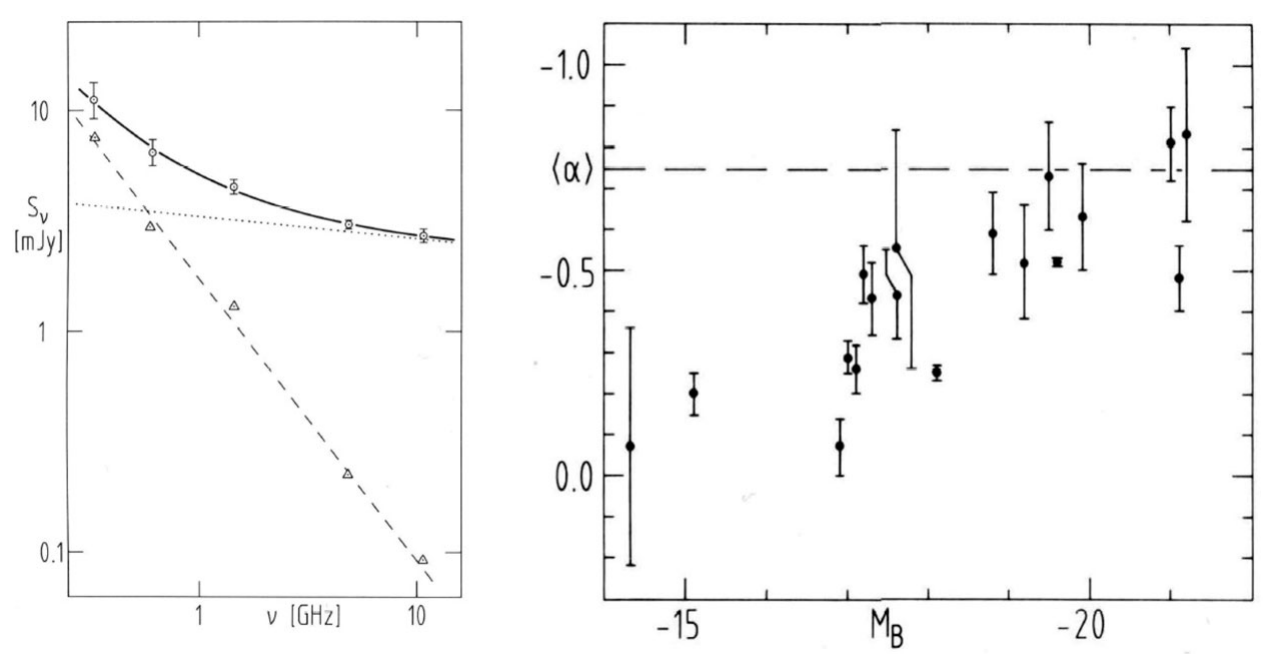

Figure 2. Left: radio continuum spectrum of the BCDG II Zw 70 , with the thermal (free-free, dotted line) and the nonthermal (synchrotron, dashed line) components indicated. The solid line represents the total flux density fitted to the measurements (from Skillman and Klein 1988). Right: spectral indices of the integrated radio emission of a sample of low-mass galaxies, mostly BCDG (from Klein et al. 1991).
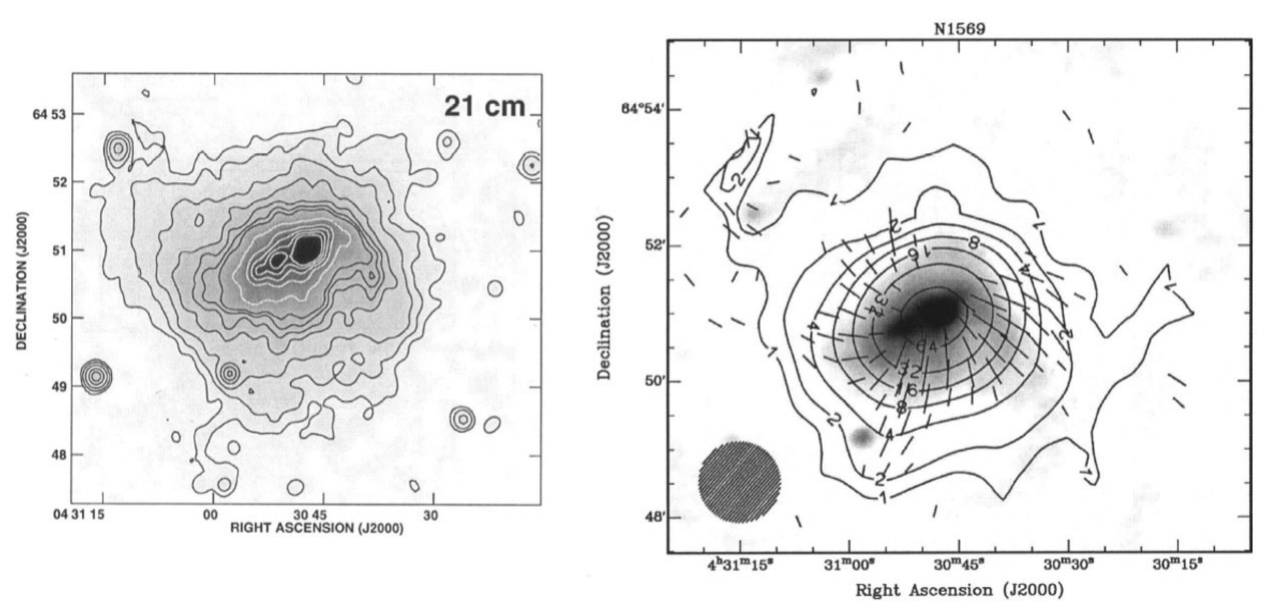

Figure 3. Synchrotron radiation of the starburst dwarf galaxy NGC 1569 (from Mühle 2003). A radio halo is visible at $21 \mathrm{~cm}$ wavelength (VLA observation, left), and the magnetic field orientation, derived from the linear polarisation at $2.8 \mathrm{~cm}$ wavelength, is primarily oriented radially outward (measurements with the Effelsberg 100-m telescope, right).

The transport of a relativistic plasma out of this galaxy is strongly suggested by two observations (Mühle 2003). First, NGC 1569 possesses a radio halo, extending out to about $2 \mathrm{kpc}$ at $1.4 \mathrm{GHz}$ (see Fig. 3, left). Second, the projected orientation of its magnetic field as deduced from measurements of the linear radio polarisation is radial throughout (Fig. 3, right). This magnetic field is just dragged along with the wind, as the energy density of the wind is about 30 times that of the magnetic field. A similar finding was made by Chyzy et al. (2000) for the dwarf irregular NGC 4449, which also possesses a low-frequency radio halo (Klein et al. 1996). 


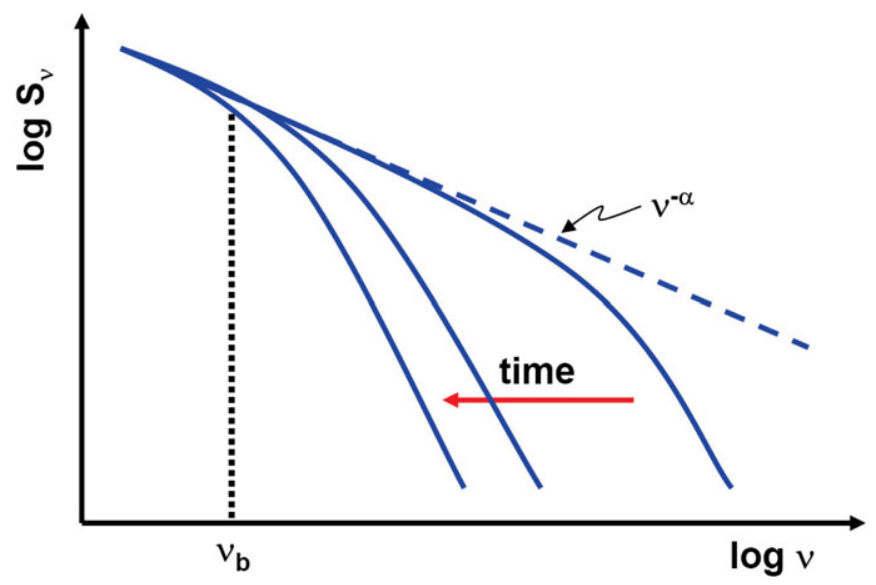

Figure 4. Temporal evolution of synchrotron spectra in galaxies.

\section{Lifetime and size of radio halos}

The lifetime of low-frequency halos around dwarf galaxies can be estimated using Eq. (2.1). With $\mu \mathrm{G}$ magnetic fields in their surroundings the dominant loss mechanism will be inverse Compton, the lifetime of relativistic electrons radiating at $120 \mathrm{MHz}$ then being of order $500 \mathrm{Myr}$, much longer than for those seen at $\mathrm{cm}$ wavelengths. This is also illustrated in Fig. 4, which sketches the temporal variation of an "aging" synchrotron spectrum. Assume that the energy spectrum of the particles initially extended to infinity. As time elapses, high-energy particles quickly lose their energy, producing a break in the spectrum. This gives rise to a corresponding break in the radiation spectrum, which wanders more and more slowly towards lower frequencies as time elapses. Hence, it takes much more time for this break to arrive at frequencies observed, e.g., with LOFAR.

While aging, particles that have left a dwarf galaxy by a wind will slowly diffuse away from it. This can happen with a maxmimum speed corresponding to the Alfvenic one, i.e.

$$
v_{A}=2.2 \cdot\left(\frac{B}{\mu G}\right) \cdot\left(\frac{n_{e}}{c m^{-3}}\right) \mathrm{km} \mathrm{s}^{-1},
$$

where $n_{e}$ is the number density of thermal electrons (or protons) of the surrounding medium. Taking $B=1 \mu \mathrm{G}$ and $n_{e}=0.001 \mathrm{~cm}^{-3}$, the relativistic particles radiating at $120 \mathrm{MHz}$ could move out to $30 \ldots 40 \mathrm{kpc}$ within $500 \mathrm{Myr}$, or correspondingly further when caught at still lower frequencies. Hence, such halos could have total sizes of $60 \ldots 80 \mathrm{kpc}$.

\section{Observations with LOFAR}

LOFAR will easily detect low-frequency halos of dwarf galaxies out to large (100 Mpc, Coma Cluster) distances. The LOFAR survey, which will cover about half the sky and will be conducted within a year's time, will provide a 5 - $\sigma$ flux limit of $0.1 \mathrm{mJy} / \mathrm{b}$.a. at $120 \mathrm{MHz}$ in 1 hour (with a synthesized beam of $1^{\prime \prime} 3$, for long baselines). Using the synchrotron halo of NGC 4449 as a template, we can estimate the expected brightness at LOFAR frequencies, which is about $0.4 \mathrm{mJy} / \mathrm{b}$.a. at $120 \mathrm{MHz}$. Its detection should hence be feasible. Assuming a $60 \mathrm{kpc}$ diameter as estimated above and placing it at $D \approx 100 \mathrm{Mpc}$ it would be seen with a $\sim 2^{\prime}$ angular extent. In a cluster or group environment, such halos are likely to trail behind the galaxy as it moves in the cluster potential with speeds much larger than the diffusion speed of the relativistic particles. This will actually provide some 
information on the proper motion of dwarf galaxies having undergone star formation over the past $10^{9} \mathrm{yrs}$. An impressive example of such trailing low-frequency radio structures is seen in the Perseus Cluster, where the head-tail radio source NGC 1265 leaves behind a huge radio tail that can be traced over a projected path of $1.1 \mathrm{Mpc}$ at low frequencies (Sijbring and de Bruyn 1998).

So, low-frequency radio emission "memorises" starburst activity up to about 1 Gyr after its termination, a time scale much longer than that of any other integral property of galaxies ( $\mathrm{H} \alpha$ and FIR luminosity, or broad-band optical/UV/IR colours). This emission therefore also provides a powerful diagnostic tool to search for post-starburst galaxies and to explore their recent-to-past star-formation rate (SFR) and magnetic field evolution.

In this context, LOFAR will also deliver important new information to address the long-standing question pertaining to possible evolutionary links between the two main classes of late-type dwarf galaxies, dwarf irregulars (dIs) and blue compact dwarf galaxies (BCDGs). BCDGs in a post-starburst phase will be easily identifiable by their weak radio emission at $\mathrm{cm}$ wavelengths in conjunction with their bright radio halos at meter wavelengths. By contrast, non-starbursting late-type dwarf galaxies will be rendered undetectable at $\mathrm{cm}$ wavelengths, while their low-frequency radio continuum emission should still exhibit the synchrotron halos created during past activity phases. Hence, if the standard evolutionary scenario, i.e. from BCDGs to dIs, is correct, then LOFAR will discover a large population of quiescent dIs with low-frequency radio halos.

\section{References}

Bertone, S., Enßlin, \& Vogt, C. 2006, MNRAS, 370, 319

Bird, J., Martini, P., \& Kaiser, C. 2008, ApJ, 676, 147

Chyzy, K. T., Beck, R., Kohle, S., Klein, U., \& Urbanik, M. 2000, A\& A, 355, 128

Clarke, T. E., Kronberg, P. P. \& Böhringer, H. 2001, ApJ, 547, 111

della Ceca, R., Griffiths, R. E., Heckman, T. M., \& MacKenty, J. W. 1996, ApJ, 469, 662

Gioia, I.M., Gregorini, L., \& Klein, U. 1982, A\&A, 116, 164

Giovannini, G., Feretti, L., Venturi, T., Kim, K.-T., \& Kronberg, P. P. 1993, ApJ, 406, 399

Klein, U. 1982, A\&A, 116, 175

Klein, U., Weiland, H., \& Brinks, E. 1991, A\& $A, 246,323$

Klein, U., Hummel, E., Bomans, D.J., \& Hopp, U. 1996, A\&્A, 313, 396

Kronberg, P. P., Lesch, H., \& Hopp, U. 1999, ApJ, 511, 56

Martin, C. L. 1998, ApJ, 506, 222

Martin, C. L. 1999, ApJ, 513, 156

Mühle, S. 2003, Ph.D. thesis, Univ. Bonn

Röttgering, H., Snellen, I., Miley, G., de Jong, J. P., Hanisch, R. J., \& Perley, R. 1994, ApJ, 436,654

Sijbring, D., \& de Bruyn, A. G. 1998, A\&A, 331, 901

Skillman, E. D., \& Klein, U. 1988, A\&AA, 199, 61 